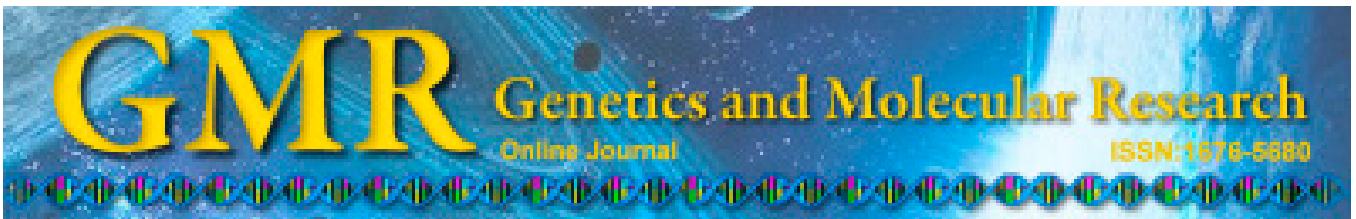

\title{
Value of human brain natriuretic peptide in treatment of acute anterior myocardial infarction evaluated via three-dimensional speckle tracking imaging
}

\author{
Y. Zhang ${ }^{1,2}$, F. Gao ${ }^{1,2}$, X.-Y. Zhang, ${ }^{1,2}$, Y.-M. Hou ${ }^{1,2}$, Y. Tang ${ }^{3}$, S.-L. Yang ${ }^{3}$, \\ S.-H. Zhu ${ }^{3}$, R.-Y. Luo ${ }^{3}$ and S.-F. Lin $^{3}$ \\ ${ }^{1}$ The Third Clinical Medical College of Southern Medical University, \\ Guangzhou, China \\ ${ }^{2}$ Department of Cardiology, Affiliated Fengxian Hospital of Southern Medical \\ University, Shanghai, China \\ ${ }^{3}$ Department of Cardiology, Affiliated Ningde Hospital of Fujian Medical \\ University Ningde, China \\ Corresponding author: Y.-M. Hou \\ E-mail: houym126vip@126.com
}

Genet. Mol. Res. 14 (2): 5699-5709 (2015)

Received May 14, 2014

Accepted October 7, 2014

Published May 29, 2015

DOI http://dx.doi.org/10.4238/2015.May.29.2

\begin{abstract}
Three-dimensional ultrasound speckle tracking imaging was used to evaluate the effects of recombinant human brain natriuretic peptide (rhBNP) in acute anterior and extensive anterior myocardial infarction. Ninety patients with acute anterior or extensive myocardial infarction were randomly divided into 3 groups: Group A [emergency percutaneous coronary intervention (PCI)], Group B (emergency PCI + rhBNP early treatment), and Group C (emergency PCI + late rhBNP treatment). Within $6 \mathrm{~h}$ of admission and at 1 week and 3 and 6 months after PCI, patients underwent routine transthoracic echocardiography and real-time three-dimensional echocardiography. At 1 week, 1 month, 3 months, 6 months, and 12 months, ejection fraction values in groups $\mathrm{B}$ and $\mathrm{C}$ were significantly greater than those in group $\mathrm{A}(\mathrm{P}<0.05)$, and
\end{abstract}


left ventricular end-diastolic volume and left ventricular end-systolic volume values in groups $\mathrm{B}$ and $\mathrm{C}$ were less than those in group $\mathrm{A}(\mathrm{P}<$ $0.05)$. Within $6 \mathrm{~h}$ of admission in each group, long-axis, radial, circumferential, and area variables corresponding to anterior descending artery segments showed no significant difference (all $\mathrm{P}>0.05$ ). However, at 1 week, 1 month, 3 months, 6 months, and 12 months, long-axis, radial, circumferential and area variables in groups $\mathrm{B}$ and $\mathrm{C}$ were significantly less than those in group A $(\mathrm{P}<0.05)$. Intervention with rhBNP can improve resilience of the local myocardium, left ventricular mechanical function, and cardiac remodeling. Within $6 \mathrm{~h}$ of admission or after PCI, rhBNP application showed no significant difference in heart function improvement or myocardial remodeling inhibition.

Key words: Myocardial infarction; Echocardiography; Three-dimensional speckle tracking imaging technology; Ventricular function; Recombinant human brain natriuretic peptide

\section{INTRODUCTION}

Acute myocardial infarction (AMI), particularly clinically common anterior myocardial infarction, is an extremely destructive acute injury to the structure and function of the heart and may be complicated by subsequent heart failure. Within a certain time window, reperfusion therapy can open an infarct-related artery and salvage ischemic myocardium to improve patient outcome. However, early recanalization does not completely inhibit ventricular remodeling. Three-dimensional ultrasound speckle tracking imaging (3D-STI) is a new technology for evaluation of cardiac structure and function that can analyze a three-dimensional full volume image of the heart and accurately evaluate myocardial movement and deformation (Helle-Valle et al., 2005). In the present study, 3D-STI technology was used in AMI patients treated with primary percutaneous cardiac intervention (PCI). In addition to conventional treatment, recombinant human brain natriuretic peptide (rhBNP) was administered, and a long-term follow-up study was conducted to evaluate the further inhibition of left ventricular remodeling and improvement of left ventricular function.

\section{MATERIAL AND METHODS}

\section{Subjects}

Between June 2011 and December 2012 in the Department of Cardiology of affiliated Ningde City Hospital of Fujian Medical University, 137 patients with the initial diagnosis of acute ST-segment elevation anterior or extensive anterior myocardial infarction were enrolled. Inclusion criteria were as follows: 1) Continuously unrelieved chest pain $>30 \mathrm{~min}$, nonresponsive to oral nitroglycerin; 2) ST-segment elevation of at least two adjacent ECG chest leads $>0.2$ $\mathrm{mV}$; 3) First AMI onset; 4) AMI onset $\leq 12 \mathrm{~h}$; 5) Infarct-related coronary artery confirmed via emergency coronary angiography to be $3 \mathrm{~cm}$ within the proximal left anterior descending artery and successfully treated with direct emergency PCI; and 6) Written informed consent obtained.

Exclusion criteria were as follows: 1) Systolic blood pressure $<90 \mathrm{mmHg}$; 2) Cardio- 
genic shock, hypovolemia, or any other clinical situation in which vasodilators would be contraindicated; 3) Severe hepatic or renal insufficiency; 4) Valvular or pulmonary heart disease; 5) Post-AMI mechanical complications: 6) Infection, trauma, cancer, or connective tissue disease; and 7) Allergy to rhBNP. One hundred and nine patients (67 males and 42 females, ages 42-78 years, mean age $61.2 \pm 14.9)$ met the inclusion criteria and signed the informed consents. Patients were divided into Group A $(\mathrm{N}=36)$, Group B $(\mathrm{N}=38)$, and Group C $(\mathrm{N}=$ 35 ) according to the random number table method. Intraoperative death occurred in 1 patient (group A), 2 patients had cardiogenic shock (group A), 1 patient had coronary perforation (group B), 1 patient had no significant coronary artery stenosis (group B), and 6 patients had poor image quality that could not be analyzed ( 2 in group A, 3 in group B, 1 in group C). Therefore, 11 patients were excluded from this study. Ultimately, 98 patients were included in the study. The basic clinical conditions of patients in the 3 groups were balanced and comparable, and differences in door-to-balloon time, cardiac function (Killip) classification, and N-terminal prohormone of brain natriuretic peptide among the 3 groups were not statistically significant $(\mathrm{P}>0.05)$ (Table 1).

\section{Table 1. Clinical Information Group.}

\begin{tabular}{lccc}
\hline Parameters & Group A $(\mathrm{N}=31)$ & Group B $(\mathrm{N}=33)$ & Group C $(\mathrm{N}=34)$ \\
\hline Gender (male/female) & $20 / 11$ & $23 / 10$ & $25 / 9$ \\
Age (years) & $60.5 \pm 16.1$ & $58.6 \pm 18.3$ & $61.1 \pm 15.7$ \\
Hypertension (N) & 24 & 21 & 23 \\
Diabetes (N) & 9 & 8 & 11 \\
High density lipoprotein hyperlipidemia (N) & 27 & $138.9 \pm 25.4$ & 31 \\
Systolic blood pressure (mmHg) & $143.8 \pm 32.7$ & $96.3 \pm 30.5$ & $140.6 \pm 25.0$ \\
Diastolic blood pressure (mmHg) & $93.5 \pm 26.6$ & $22.5 \pm 18.01$ & $94.2 \pm 31.1$ \\
Smoking history (years) & $21.6 \pm 13.7$ & $8.3 \pm 3.7$ & $21.5 \pm 21.5$ \\
Onset to treatment time (h) & $7.3 \pm 2.6$ & $77.3 \pm 12.8$ & $6.5 \pm 4.6$ \\
Croquet time (min) & $67.3 \pm 13.6$ & $2.4 \pm 0.9$ & $74.4 \pm 13.7$ \\
Killips classification & $2.1 \pm 1.2$ & $2.5 \pm 1.3$ \\
\hline
\end{tabular}

\section{Treatment}

In Group A, patients were routinely given aspirin $300 \mathrm{mg}$ plus clopidogrel $300 \mathrm{mg}$ orally, successful emergency PCI treatment was performed, thrombolysis in myocardial infarction (TIMI) was grade III, and conventional therapy was administered after PCI. In Group B, within $6 \mathrm{~h}$ of admission, patients were routinely given aspirin $300 \mathrm{mg}$ plus clopidogrel $300 \mathrm{mg}$ orally as well as a loading dose of rhBNP (Trademark: XinHuoSu, Chengdu Nuodikang Biopharmaceutical Co., Ltd.) $1.5 \mu \mathrm{g} / \mathrm{kg}$ intravenously. The intravenous infusion was maintained for $72 \mathrm{~h}$ at $0.0075 \mu \mathrm{g} \cdot \mathrm{kg}^{-1} \cdot \mathrm{min}^{-1}$ (dose adjusted according to blood pressure), successful emergency PCI treatment was performed, TIMI flow was grade III, and the process was supplemented by conventional drug therapy. In Group C, rhBNP treatment began after the completion of emergency $\mathrm{PCI}$, and the remaining protocol was the same as for patients in Group B.

\section{Instruments and methods}

1) Image acquisition: Images were obtained within $6 \mathrm{~h}$ of admission and at 1 week, 1 month, 3 months, 6 months, and 12 months after PCI. The Vivid E9 ultrasonic diagnostic apparatus was used (GE Healthcare, USA), and an ECG was simultaneously recorded. Subjects 
were examined in the left lateral position with calm respiratory status. A conventional twodimensional probe (frequency 1-5 MHz) was used to acquire long-axis and short-axis views of the parasternal left ventricle; apical four-chamber, two-chamber, and three-chamber heart; and other facet images.

2) Conventional echocardiographic parameters: After the three-dimensional image acquisition was completed, the same observer reacquired images of two-dimensional apical four-chamber and parasternal left ventricular long-axis views. The two-dimensional echocardiography biplane modified Simpson method was used to measure left ventricular end-diastolic volume (LVEDV), left ventricular end-systolic volume (LVESV), and ejection fraction (EF) values, and 3 consecutive measurements were averaged.

3) A three-dimensional volume probe (frequency 1-5 MHz) was switched on to detect the apical four-chamber image. The pre-four-dimensional mode was selected to adjust the image of the left ventricle intima clearly, and the full volume four-dimensional mode was then entered. Subjects held their breath at the end of expiration. The imaging angle was $\left(70-80^{\circ}\right) \times\left(70-80^{\circ}\right)$, and a threedimensional full-volume dynamic cardiac map was obtained and stored.

4) Image analysis: the EchoPac PC 2011 software (GE Healthcare, USA) was used, and the image data was stored for offline analysis. 3D-STI was performed to analyze the full volume image of the apex. Software can automatically cut and generate multiple facet images from the end-diastolic full volume image. The position of the cut line was adjusted to get images of the apical four-chamber, two-chamber, and left ventricular long axis so that the position of the apex and the left ventricular bottom could be determined.

The software automatically depicts the left ventricular endocardial border, but it can be manually adjusted if necessary. The location of echo spots should be confirmed within the myocardium. The software will automatically calculate left ventricular EF and track motion information in three-dimensional space in the myocardial region of interest. The left ventricle is divided into 17 segments, including 3 anterior segments [basal anterior (BA), median anterior (MA), apex anterior (AA)], 5 septal wall segments [basal anterior septal (BAS), median anterior septal (MAS), basal inferior septal (BIS), median inferior septal (MIS), apex septal (AS)], 3 inferior wall segments [basal inferior (BI), median inferior (MI), apex inferior (AI)], 5 lateral wall segments [basal anterior lateral (BAL), median anterior lateral (MAL), basal inferior lateral (BIL), median inferior lateral (MIL), apex lateral (AL), and apical (A)]. Longitudinal (the major axis direction), radial (short-axis direction), circumferential, and area variables of the 17 segments were calculated.

\section{Statistical Analysis}

The above indicators were all numeric variables. Excel and the SPSS 16.0 statistical analysis software were used to establish the appropriate database for parallel statistical analysis. Measurement data are reported as means $\pm \mathrm{SD}$, and analysis of variance (ANOVA) was used to compare the difference among groups. $\mathrm{P}<0.05$ was considered to be statistically significant.

\section{RESULTS}

\section{Conventional echocardiographic parameters of different groups}

Within $6 \mathrm{~h}$ after admission, EF values of each group had no significant difference 
(all $\mathrm{P}>0.05$ ). With the follow-up period extended to 1 week, 1 month, 3 months, 6 months, and 12 months, the EF values in Groups B and C were greater than those in Group A, and the difference was statistically significant $(\mathrm{P}<0.05)$. No significant difference was seen between Groups B and C $(P>0.05)$ (Figure 1). Similarly, within $6 \mathrm{~h}$ of admission, LVEDV and LVESV of each group showed no significant difference (all $\mathrm{P}>0.05$ ). When the follow-up time was extended to 1 week after PCI, EF values in Groups B and C were less than those in Group A, and the difference was statistically significant $(P<0.05)$. No significant difference was seen between Groups B and C $(\mathrm{P}>0.05)$ (Figures 2 and 3).

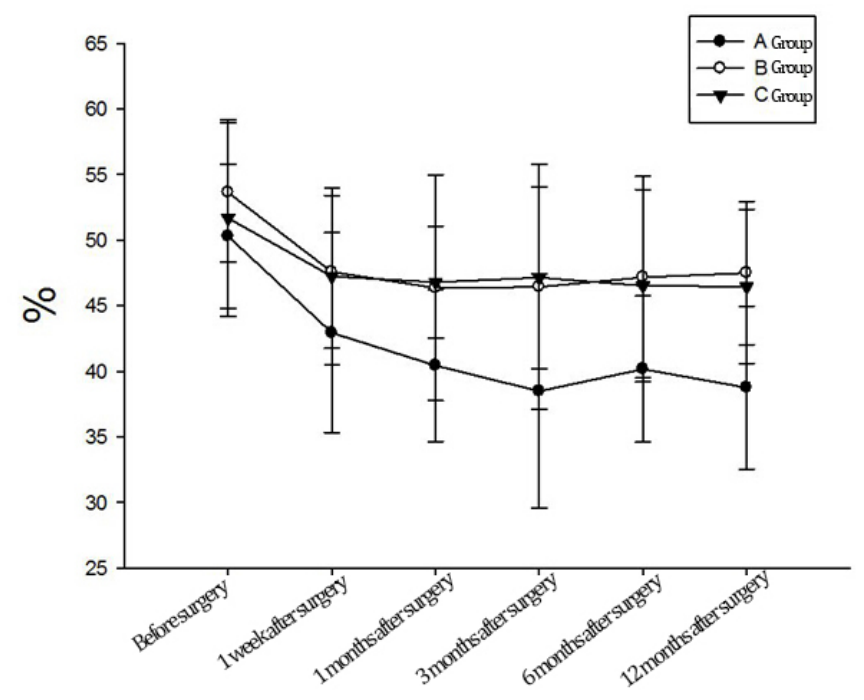

Figure 1. Value of each group dynamic changes of left ventricular EF.

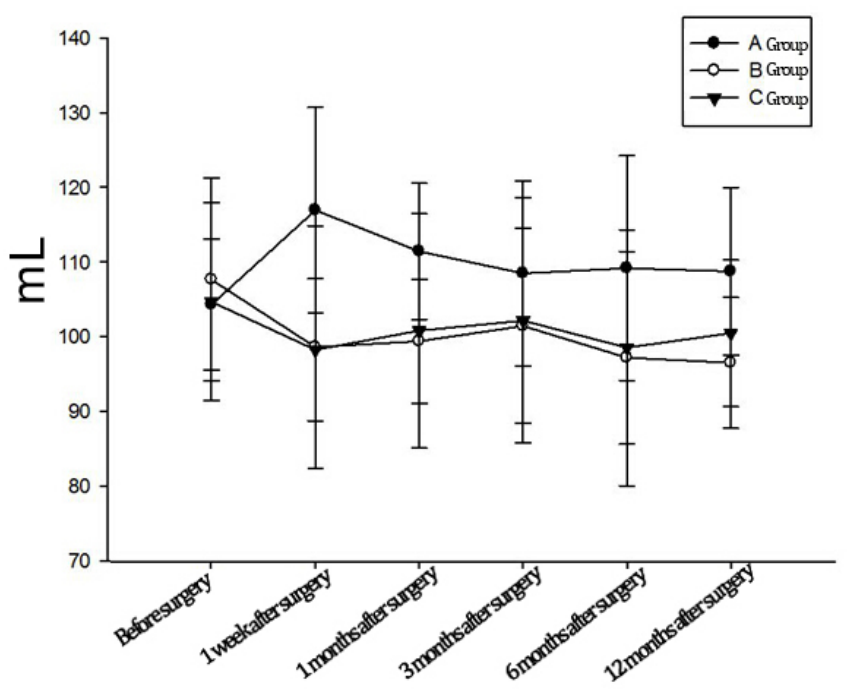

Figure 2. LVEDV changes in each group. 


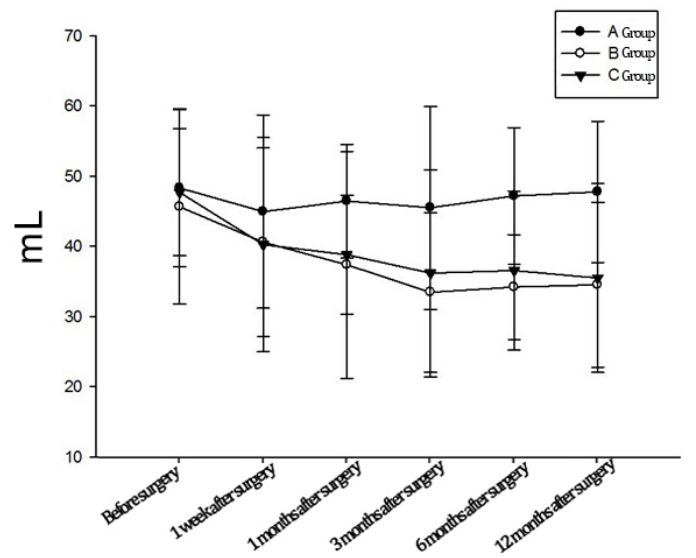

Figure 3. LVESV changes in each group.

\section{Corresponding 3D-STI test results of each segment of the left ventricular wall in different groups}

1) Long-axis variables: Within $6 \mathrm{~h}$ after admission, the long-axis variables corresponding to anterior descending artery segments in each group showed no significant differences (all $\mathrm{P}>0.05$ ). With prolonged follow-up at 1 month, 6 months, and 12 months, the long-axis variables in Groups B and C were less than those in Group A, and the difference was statistically significant $(\mathrm{P}<0.05)$. However, no statistically significant difference was seen between Groups B and C (all P > 0.05) (Table 2).

\begin{tabular}{|c|c|c|c|c|c|c|c|}
\hline & & $\begin{array}{c}\text { Within } 6 \mathrm{~h} \\
\text { of admission }\end{array}$ & $\begin{array}{l}1 \text { week after } \\
\text { surgery }\end{array}$ & $\begin{array}{l}1 \text { months after } \\
\text { surgery }\end{array}$ & $\begin{array}{l}3 \text { months after } \\
\text { surgery }\end{array}$ & $\begin{array}{c}6 \text { months after } \\
\text { surgery }\end{array}$ & $\begin{array}{l}12 \text { months } \\
\text { after surgery }\end{array}$ \\
\hline \multirow[t]{7}{*}{ Group A } & BA & $7.21 \pm 1.63$ & $8.86 \pm 2.33$ & $8.24 \pm 2.73$ & $7.35 \pm 2.05$ & $9.53 \pm 2.12$ & $8.30 \pm 2.59$ \\
\hline & BAS & $8.36 \pm 1.74$ & $8.78 \pm 3.16$ & $7.75 \pm 2.09$ & $9.02 \pm 2.52$ & $6.90 \pm 3.01$ & $7.11 \pm 2.46$ \\
\hline & MA & $11.12 \pm 2.67$ & $9.42 \pm 2.84$ & $8.62 \pm 2.54$ & $10.51 \pm 2.37$ & $9.63 \pm 3.52$ & $9.37 \pm 3.83$ \\
\hline & MAS & $8.43 \pm 1.57$ & $9.03 \pm 4.47$ & $9.20 \pm 2.42$ & $9.16 \pm 5.01$ & $10.34 \pm 2.68$ & $10.67 \pm 2.52$ \\
\hline & AA & $8.36 \pm 3.74$ & $7.78 \pm 3.04$ & $7.75 \pm 4.09$ & $8.76 \pm 2.59$ & $6.90 \pm 3.76$ & $7.53 \pm 2.27$ \\
\hline & AS & $8.62 \pm 2.02$ & $9.26 \pm 2.13$ & $9.77 \pm 2.57$ & $9.36 \pm 2.70$ & $9.46 \pm 2.66$ & $9.92 \pm 2.16$ \\
\hline & A & $9.16 \pm 2.04$ & $10.57 \pm 3.62$ & $12.45 \pm 2.68$ & $10.34 \pm 2.41$ & $11.40 \pm 3.25$ & $10.11 \pm 2.59$ \\
\hline \multirow[t]{7}{*}{ Group B } & $\mathrm{BA}$ & $8.53 \pm 2.78$ & $10.55 \pm 2.51$ & $14.64 \pm 2.95 *$ & $17.36 \pm 2.30 *$ & $16.62 \pm 2.67 *$ & $17.53 \pm 3.10 *$ \\
\hline & BAS & $9.15 \pm 2.03$ & $10.03 \pm 2.35$ & $13.78 \pm 2.32 *$ & $15.26 \pm 2.32 *$ & $16.59 \pm 3.64 *$ & $15.32 \pm 4.87^{*}$ \\
\hline & MA & $8.95 \pm 3.45$ & $10.45 \pm 3.63$ & $14.15 \pm 3.65^{*}$ & $15.44 \pm 2.62 *$ & $16.05 \pm 3.48^{*}$ & $17.37 \pm 3.61^{*}$ \\
\hline & MAS & $8.47 \pm 3.35$ & $9.65 \pm 2.68$ & $14.76 \pm 2.57 *$ & $16.75 \pm 2.01^{*}$ & $17.64 \pm 2.69^{*}$ & $16.21 \pm 3.04^{*}$ \\
\hline & AA & $8.31 \pm 3.25$ & $8.17 \pm 3.27$ & $12.45 \pm 2.80 *$ & $14.41 \pm 4.12 *$ & $14.00 \pm 3.94 *$ & $15.61 \pm 3.42 *$ \\
\hline & AS & $8.57 \pm 2.86$ & $9.05 \pm 2.16$ & $14.34 \pm 2.35^{*}$ & $15.66 \pm 2.93 *$ & $16.21 \pm 3.76^{*}$ & $16.36 \pm 3.56^{*}$ \\
\hline & A & $9.43 \pm 2.73$ & $10.37 \pm 2.18$ & $14.60 \pm 2.84^{*}$ & $17.28 \pm 3.05^{*}$ & $16.26 \pm 2.63^{*}$ & $16.32 \pm 3.48^{*}$ \\
\hline \multirow[t]{7}{*}{ Group C } & BA & $7.89 \pm 2.15$ & $8.04 \pm 2.65$ & $13.06 \pm 2.53^{*}$ & $16.17 \pm 4.15^{*}$ & $15.42 \pm 2.81^{*}$ & $16.77 \pm 2.85^{*}$ \\
\hline & BAS & $8.33 \pm 2.17$ & $7.75 \pm 3.48$ & $13.55 \pm 2.97 *$ & $18.63 \pm 2.91 *$ & $16.06 \pm 3.68^{*}$ & $17.36 \pm 2.78^{*}$ \\
\hline & MA & $8.26 \pm 4.28$ & $9.65 \pm 2.37$ & $15.70 \pm 2.57 *$ & $16.64 \pm 2.01 *$ & $17.63 \pm 2.68^{*}$ & $16.23 \pm 2.63^{*}$ \\
\hline & MAS & $9.46 \pm 2.45$ & $10.74 \pm 3.23$ & $12.15 \pm 4.86$ & $14.37 \pm 2.49^{*}$ & $14.46 \pm 3.57 *$ & $14.17 \pm 2.93^{*}$ \\
\hline & AA & $8.32 \pm 2.85$ & $10.15 \pm 2.16$ & $15.47 \pm 2.53^{*}$ & $17.61 \pm 2.38^{*}$ & $16.21 \pm 2.75^{*}$ & $17.30 \pm 3.04 *$ \\
\hline & AS & $9.24 \pm 2.76$ & $10.05 \pm 2.58$ & $13.84 \pm 2.27 *$ & $15.63 \pm 2.28^{*}$ & $16.90 \pm 3.44^{*}$ & $15.23 \pm 2.75^{*}$ \\
\hline & A & $8.53 \pm 3.56$ & $9.49 \pm 3.33$ & $16.53 \pm 3.15^{*}$ & $15.41 \pm 2.22 *$ & $16.54 \pm 3.83^{*}$ & $16.75 \pm 4.12^{*}$ \\
\hline
\end{tabular}

The table strain values are negative; BA: at the base of the front wall, BAS: near to the wall of the base next door, MA: central anterior, MAS: central near to intramural next door, AA: apex anterior, AS: inter-apical next door, A: apex; compared with group $\mathrm{A},{ }^{*} \mathrm{P}<0.05$. 
2) Radial variables: Within $6 \mathrm{~h}$ after admission, the short-axis variables corresponding to anterior descending artery segments in each group showed no significant differences (all $\mathrm{P}>$ $0.05)$. With prolonged follow-up at 1 month, 6 months, and 12 months, the short-axis variables in Groups B and C were less than those in Group A, and the difference was statistically significant $(\mathrm{P}<0.05)$. However, no statistically significant difference was seen between Groups $\mathrm{B}$ and $\mathrm{C}($ all $\mathrm{P}>0.05)$ (Tables 2 and 3).

\begin{tabular}{|c|c|c|c|c|c|c|c|}
\hline & & $\begin{array}{l}\text { Within } 6 \mathrm{~h} \\
\text { of admission }\end{array}$ & $\begin{array}{c}1 \text { week after } \\
\text { surgery }\end{array}$ & $\begin{array}{l}1 \text { month after } \\
\text { surgery }\end{array}$ & $\begin{array}{c}3 \text { months after } \\
\text { surgery }\end{array}$ & $\begin{array}{c}6 \text { months after } \\
\text { surgery }\end{array}$ & $\begin{array}{c}12 \text { months after } \\
\text { surgery }\end{array}$ \\
\hline \multirow[t]{7}{*}{ Group A } & BA & $18.53 \pm 5.58$ & $18.46 \pm 4.56$ & $20.53 \pm 5.35$ & $19.56 \pm 4.32$ & $20.77 \pm 4.46$ & $21.31 \pm 4.16$ \\
\hline & BAS & $18.43 \pm 4.60$ & $19.2 \pm 4.63$ & $19.42 \pm 4.67$ & $19.36 \pm 4.65$ & $21.42 \pm 4.50$ & $20.63 \pm 4.85$ \\
\hline & MA & $19.65 \pm 4.37$ & $19.52 \pm 4.76$ & $21.05 \pm 2.00$ & $19.23 \pm 6.19$ & $21.15 \pm 4.35$ & $20.36 \pm 5.52$ \\
\hline & MAS & $19.45 \pm 4.65$ & $20.34 \pm 4.25$ & $20.80 \pm 4.37$ & $20.64 \pm 4.25$ & $19.60 \pm 7.07$ & $19.25 \pm 4.89$ \\
\hline & AA & $18.92 \pm 4.56$ & $20.52 \pm 4.67$ & $21.16 \pm 4.52$ & $21.49 \pm 4.26$ & $19.52 \pm 4.59$ & $20.32 \pm 4.64$ \\
\hline & AS & $21.47 \pm 4.77$ & $22.56 \pm 6.47$ & $20.57 \pm 4.25$ & $23.01 \pm 4.97$ & $21.67 \pm 3.63$ & $22.65 \pm 3.84$ \\
\hline & A & $19.46 \pm 3.74$ & $21.45 \pm 3.53$ & $23.68 \pm 3.97$ & $21.23 \pm 5.56$ & $22.33 \pm 4.66$ & $24.31 \pm 5.16$ \\
\hline \multirow[t]{7}{*}{ Group B } & BA & $21.83 \pm 4.50$ & $27.63 \pm 4.80^{*}$ & $31.48 \pm 3.25^{*}$ & $30.26 \pm 3.23^{*}$ & $31.42 \pm 4.43 *$ & $30.64 \pm 4.86^{*}$ \\
\hline & BAS & $20.25 \pm 4.84$ & $26.06 \pm 3.57 *$ & $30.15 \pm 4.35^{*}$ & $30.42 \pm 4.36^{*}$ & $29.76 \pm 4.15^{*}$ & $31.26 \pm 4.53^{*}$ \\
\hline & MA & $19.36 \pm 4.49$ & $28.34 \pm 4.38^{*}$ & $32.64 \pm 4.86^{*}$ & $28.37 \pm 5.49^{*}$ & $28.66 \pm 4.56^{*}$ & $29.57 \pm 4.96^{*}$ \\
\hline & MAS & $20.46 \pm 4.45$ & $28.35 \pm 4.52 *$ & $31.63 \pm 3.93^{*}$ & $30.64 \pm 4.85^{*}$ & $30.46 \pm 4.70^{*}$ & $32.35 \pm 4.17^{*}$ \\
\hline & AA & $19.36 \pm 4.88$ & $26.13 \pm 4.97^{*}$ & $28.78 \pm 4.39 *$ & $30.17 \pm 6.38^{*}$ & $29.28 \pm 7.74 *$ & $30.38 \pm 5.64 *$ \\
\hline & AS & $19.21 \pm 4.38$ & $26.70 \pm 5.07 *$ & $29.44 \pm 4.11^{*}$ & $32.65 \pm 4.62 *$ & $30.35 \pm 4.63^{*}$ & $31.75 \pm 4.50^{*}$ \\
\hline & A & $21.14 \pm 4.50$ & $26.45 \pm 4.86^{*}$ & $29.37 \pm 4.44^{*}$ & $31.36 \pm 4.15^{*}$ & $29.57 \pm 3.96^{*}$ & $32.56 \pm 4.86^{*}$ \\
\hline \multirow[t]{7}{*}{ Group C } & BA & $22.31 \pm 5.75$ & $28.47 \pm 4.54^{*}$ & $31.61 \pm 4.35^{*}$ & $30.31 \pm 4.55^{*}$ & $30.31 \pm 4.46^{*}$ & $29.43 \pm 4.59^{*}$ \\
\hline & BAS & $18.34 \pm 4.64$ & $27.46 \pm 4.55^{*}$ & $30.74 \pm 4.26^{*}$ & $31.35 \pm 4.61^{*}$ & $31.25 \pm 3.67^{*}$ & $28.68 \pm 4.57^{*}$ \\
\hline & MA & $19.43 \pm 7.78$ & $28.45 \pm 4.80^{*}$ & $30.51 \pm 4.52^{*}$ & $30.46 \pm 3.42 *$ & $32.63 \pm 3.54^{*}$ & $32.65 \pm 3.83^{*}$ \\
\hline & MAS & $20.22 \pm 4.65$ & $29.46 \pm 4.05^{*}$ & $27.65 \pm 4.96^{*}$ & $32.53 \pm 3.23^{*}$ & $30.33 \pm 3.13^{*}$ & $31.34 \pm 4.59^{*}$ \\
\hline & AA & $19.14 \pm 6.07$ & $26.00 \pm 5.35^{*}$ & $29.58 \pm 5.32 *$ & $31.06 \pm 5.73^{*}$ & $30.19 \pm 4.11^{*}$ & $31.27 \pm 6.46^{*}$ \\
\hline & AS & $19.98 \pm 5.43$ & $28.43 \pm 4.68^{*}$ & $27.95 \pm 4.05^{*}$ & $30.94 \pm 5.82^{*}$ & $30.55 \pm 3.48^{*}$ & $30.37 \pm 4.65^{*}$ \\
\hline & A & $20.35 \pm 4.83$ & $27.45 \pm 4.16^{*}$ & $28.37 \pm 4.33^{*}$ & $32.64 \pm 4.58^{*}$ & $32.91 \pm 2.58^{*}$ & $32.33 \pm 4.64 *$ \\
\hline
\end{tabular}

Table strain values are negative; BA: at the base of the front wall, BAS: near to the wall of the base next door, MA: central anterior, MAS: central near to intramural next door, AA: apex anterior, AS: inter-apical next door, A: apex; compared with group $\mathrm{A}, * \mathrm{P}<0.05$.

3) Circumference variables: Within $6 \mathrm{~h}$ after admission, the circumference variables corresponding to anterior descending artery segments in each group showed no significant differences (all $\mathrm{P}>0.05$ ). With prolonged follow-up at 1 month, 6 months, and 12 months, the circumference variables in Groups B and C were less than those in Group A, and the difference was statistically significant $(\mathrm{P}<0.05)$. However, no statistically significant difference was seen between Groups B and C (all P > 0.05) (Table 4).

4) Area variables: Within $6 \mathrm{~h}$ after admission, the area variables corresponding to anterior descending artery segments in each group showed no significant differences (all $\mathrm{P}>$ 0.05). With prolonged follow-up at 1 month, 6 months, and 12 months, the area variables in Groups B and C were less than those in Group A, and the difference was statistically significant $(\mathrm{P}<0.05)$. However, no statistically significant difference was seen between Groups $\mathrm{B}$ and $\mathrm{C}$ (all $\mathrm{P}>0.05)$ (Table 5).

5) Among the 3 groups, at all time points, no difference was seen in circumflex coronary artery and right coronary artery segments (all $\mathrm{P}>0.05$ ). 
Table 4. Follow-up of each group dominated anterior descending artery myocardial segments circumferential strain (means $\pm \mathrm{SD}, \%$ ).

\begin{tabular}{|c|c|c|c|c|c|c|c|}
\hline & & $\begin{array}{c}\text { Within } 6 \mathrm{~h} \\
\text { of admission }\end{array}$ & $\begin{array}{l}1 \text { week after } \\
\text { surgery }\end{array}$ & $\begin{array}{l}1 \text { month after } \\
\text { surgery }\end{array}$ & $\begin{array}{c}3 \text { months after } \\
\text { surgery }\end{array}$ & $\begin{array}{c}6 \text { months after } \\
\text { surgery }\end{array}$ & $\begin{array}{l}12 \text { months after } \\
\text { surgery }\end{array}$ \\
\hline \multirow[t]{7}{*}{ Group A } & BA & $18.36 \pm 4.74$ & $16.66 \pm 4.43$ & $18.34 \pm 6.46$ & $19.27 \pm 4.21$ & $20.72 \pm 4.18$ & $19.03 \pm 5.25$ \\
\hline & BAS & $17.56 \pm 4.33$ & $19.24 \pm 5.73$ & $19.46 \pm 4.71$ & $19.26 \pm 5.64$ & $18.35 \pm 7.06$ & $20.30 \pm 4.83$ \\
\hline & MA & $18.25 \pm 5.15$ & $19.48 \pm 4.63$ & $21.35 \pm 5.03$ & $19.67 \pm 4.46$ & $21.14 \pm 5.37$ & $20.33 \pm 5.28$ \\
\hline & MAS & $19.33 \pm 4.78$ & $20.05 \pm 5.85$ & $21.46 \pm 4.95$ & $19.47 \pm 5.32$ & $18.23 \pm 4.60$ & $20.25 \pm 5.17$ \\
\hline & $\mathrm{AA}$ & $18.34 \pm 5.22$ & $21.35 \pm 4.32$ & $19.00 \pm 7.18$ & $20.36 \pm 4.95$ & $21.67 \pm 5.31$ & $20.36 \pm 4.37$ \\
\hline & AS & $18.73 \pm 7.56$ & $22.26 \pm 4.65$ & $20.35 \pm 4.71$ & $23.43 \pm 4.21$ & $21.84 \pm 6.67$ & $22.28 \pm 4.37$ \\
\hline & A & $19.25 \pm 4.36$ & $21.25 \pm 5.27$ & $20.85 \pm 4.96$ & $21.36 \pm 4.67$ & $22.42 \pm 4.26$ & $24.13 \pm 5.63$ \\
\hline \multirow[t]{7}{*}{ Group B } & BA & $18.83 \pm 4.16$ & $27.34 \pm 5.76^{*}$ & $31.53 \pm 5.37 *$ & $30.31 \pm 6.32 *$ & $31.23 \pm 4.94 *$ & $30.46 \pm 5.17 *$ \\
\hline & BAS & $18.47 \pm 5.32$ & $25.66 \pm 5.92 *$ & $30.33 \pm 7.85^{*}$ & $30.40 \pm 5.54 *$ & $29.40 \pm 4.25 *$ & $31.65 \pm 5.64 *$ \\
\hline & MA & $19.23 \pm 4.70$ & $26.09 \pm 4.81^{*}$ & $31.59 \pm 4.23^{*}$ & $30.46 \pm 6.36^{*}$ & $32.61 \pm 6.52 *$ & $32.15 \pm 4.81 *$ \\
\hline & MAS & $20.22 \pm 4.65$ & $27.46 \pm 4.05^{*}$ & $30.65 \pm 4.46^{*}$ & $32.53 \pm 3.29^{*}$ & $30.33 \pm 3.29 *$ & $34.14 \pm 4.57 *$ \\
\hline & $\mathrm{AA}$ & $19.65 \pm 4.36$ & $26.53 \pm 4.57^{*}$ & $31.22 \pm 4.93 *$ & $30.14 \pm 4.38^{*}$ & $30.25 \pm 4.35 *$ & $32.40 \pm 5.36^{*}$ \\
\hline & AS & $19.21 \pm 4.68$ & $29.70 \pm 5.08^{*}$ & $31.42 \pm 5.61 *$ & $32.65 \pm 4.62 *$ & $30.35 \pm 4.63 *$ & $31.75 \pm 4.54 *$ \\
\hline & A & $19.33 \pm 6.43$ & $30.31 \pm 4.34^{*}$ & $32.67 \pm 4.63^{*}$ & $34.37 \pm 5.42 *$ & $34.60 \pm 4.26^{*}$ & $34.54 \pm 4.96 *$ \\
\hline \multirow[t]{7}{*}{ Group C } & BA & $20.11 \pm 6.34$ & $29.28 \pm 7.74^{*}$ & $30.38 \pm 5.64 *$ & $30.47 \pm 4.54^{*}$ & $31.61 \pm 4.35^{*}$ & $30.31 \pm 4.55^{*}$ \\
\hline & BAS & $20.65 \pm 4.62$ & $26.35 \pm 4.63^{*}$ & $31.75 \pm 4.50 *$ & $29.46 \pm 4.55^{*}$ & $30.04 \pm 4.27 *$ & $31.35 \pm 4.61 *$ \\
\hline & MA & $19.31 \pm 5.75$ & $27.47 \pm 4.53^{*}$ & $31.61 \pm 4.36^{*}$ & $30.31 \pm 4.55$ & $30.31 \pm 4.46^{*}$ & $35.43 \pm 4.59 *$ \\
\hline & MAS & $18.60 \pm 4.34$ & $26.46 \pm 4.55^{*}$ & $30.74 \pm 4.26^{*}$ & $31.35 \pm 4.61$ & $31.25 \pm 3.67^{*}$ & $34.68 \pm 4.57 *$ \\
\hline & $\mathrm{AA}$ & $20.34 \pm 4.83$ & $27.35 \pm 4.36^{*}$ & $34.71 \pm 6.04 *$ & $31.26 \pm 4.72$ & $33.55 \pm 5.91 *$ & $32.25 \pm 4.83^{*}$ \\
\hline & AS & $18.39 \pm 5.37$ & $27.73 \pm 4.23^{*}$ & $32.45 \pm 5.91^{*}$ & $34.55 \pm 5.06^{*}$ & $34.25 \pm 4.72 *$ & $35.11 \pm 6.23^{*}$ \\
\hline & A & $20.35 \pm 4.85$ & $27.45 \pm 4.16^{*}$ & $32.37 \pm 4.33$ & $32.64 \pm 4.78^{*}$ & $32.91 \pm 2.50 *$ & $32.33 \pm 4.61$ \\
\hline
\end{tabular}

The table strain values are negative; BA: at the base of the front wall, BAS: near to the wall of the base next door, MA: central anterior, MAS: central near to intramural next door, AA: apex anterior, AS: inter-apical next door, A: apex; compared with group $\mathrm{A},{ }^{*} \mathrm{P}<0.05$.

Table 5. Follow-up of each group dominant right coronary myocardial segments area strain (means $\pm \mathrm{SD}, \%)$.

\begin{tabular}{|c|c|c|c|c|c|c|c|}
\hline & & $\begin{array}{c}\text { Within } 6 \mathrm{~h} \\
\text { of admission }\end{array}$ & $\begin{array}{c}1 \text { week after } \\
\text { surgery }\end{array}$ & $\begin{array}{l}1 \text { month after } \\
\text { surgery }\end{array}$ & $\begin{array}{c}3 \text { months after } \\
\text { surgery }\end{array}$ & $\begin{array}{c}6 \text { months after } \\
\text { surgery }\end{array}$ & $\begin{array}{c}12 \text { months after } \\
\text { surgery }\end{array}$ \\
\hline \multirow[t]{7}{*}{ Group A } & BA & $14.45 \pm 4.31$ & $14.11 \pm 4.13$ & $15.31 \pm 4.61$ & $16.57 \pm 4.72$ & $14.43 \pm 4.53$ & $15.35 \pm 4.84$ \\
\hline & BAS & $15.05 \pm 4.69$ & $16.63 \pm 4.46$ & $13.15 \pm 3.99$ & $15.86 \pm 4.18$ & $16.49 \pm 4.68$ & $15.61 \pm 4.67$ \\
\hline & MA & $15.68 \pm 4.77$ & $13.78 \pm 4.35$ & $17.10 \pm 4.12$ & $15.68 \pm 4.98$ & $16.04 \pm 3.96$ & $17.58 \pm 5.11$ \\
\hline & MAS & $15.25 \pm 5.43$ & $15.46 \pm 4.07$ & $16.53 \pm 4.63$ & $17.83 \pm 5.39$ & $16.63 \pm 4.48$ & $17.68 \pm 6.33$ \\
\hline & AA & $17.13 \pm 4.26$ & $16.31 \pm 4.28$ & $18.36 \pm 4.51$ & $16.06 \pm 4.32$ & $17.16 \pm 4.62$ & $18.93 \pm 4.67$ \\
\hline & AS & $15.43 \pm 5.52$ & $14.85 \pm 4.24$ & $15.15 \pm 4.29$ & $14.25 \pm 4.16$ & $14.50 \pm 5.17$ & $15.26 \pm 4.34$ \\
\hline & A & $15.15 \pm 4.15$ & $16.57 \pm 4.16$ & $14.56 \pm 4.80$ & $14.34 \pm 5.03$ & $16.43 \pm 3.85$ & $15.01 \pm 4.96$ \\
\hline \multirow[t]{7}{*}{ Group B } & $\mathrm{BA}$ & $15.56 \pm 4.67$ & $24.55 \pm 3.43^{*}$ & $24.37 \pm 4.66^{*}$ & $24.15 \pm 4.33^{*}$ & $26.61 \pm 5.67^{*}$ & $27.33 \pm 4.14^{*}$ \\
\hline & BAS & $14.78 \pm 4.18$ & $23.03 \pm 4.85^{*}$ & $23.84 \pm 4.17^{*}$ & $25.67 \pm 4.38^{*}$ & $23.54 \pm 4.61 *$ & $25.38 \pm 4.87^{*}$ \\
\hline & MA & $16.53 \pm 2.78$ & $20.45 \pm 5.56^{*}$ & $24.00 \pm 5.95^{*}$ & $27.16 \pm 5.20^{*}$ & $26.12 \pm 5.67^{*}$ & $27.37 \pm 5.01 *$ \\
\hline & MAS & $15.11 \pm 4.15$ & $26.45 \pm 4.13 *$ & $24.56 \pm 3.65^{*}$ & $25.41 \pm 4.16^{*}$ & $26.05 \pm 4.48^{*}$ & $27.77 \pm 3.61 *$ \\
\hline & AA & $16.56 \pm 4.88$ & $24.65 \pm 4.46^{*}$ & $24.69 \pm 4.54^{*}$ & $26.58 \pm 4.01^{*}$ & $27.64 \pm 4.65^{*}$ & $26.16 \pm 4.46^{*}$ \\
\hline & AS & $17.24 \pm 4.25$ & $24.56 \pm 5.52 *$ & $25.17 \pm 4.96^{*}$ & $24.12 \pm 4.06^{*}$ & $26.35 \pm 4.58^{*}$ & $27.64 \pm 5.51^{*}$ \\
\hline & A & $15.11 \pm 4.14$ & $24.17 \pm 5.16^{*}$ & $23.45 \pm 4.05^{*}$ & $24.45 \pm 4.16^{*}$ & $24.00 \pm 4.94 *$ & $25.31 \pm 6.14 *$ \\
\hline \multirow[t]{7}{*}{ Group C } & BA & $17.08 \pm 5.15$ & $24.41 \pm 4.14^{*}$ & $24.68 \pm 4.48^{*}$ & $26.17 \pm 4.57^{*}$ & $24.63 \pm 4.54 *$ & $26.34 \pm 5.14 *$ \\
\hline & BAS & $14.53 \pm 4.23$ & $24.03 \pm 4.76^{*}$ & $25.07 \pm 4.11 *$ & $25.16 \pm 4.35^{*}$ & $24.34 \pm 4.61 *$ & $24.68 \pm 4.52 *$ \\
\hline & MA & $15.56 \pm 6.38$ & $20.65 \pm 5.58^{*}$ & $24.16 \pm 4.95^{*}$ & $27.47 \pm 4.43^{*}$ & $26.61 \pm 4.35^{*}$ & $27.34 \pm 4.06^{*}$ \\
\hline & MAS & $15.94 \pm 4.85$ & $24.00 \pm 4.91 *$ & $27.58 \pm 4.97^{*}$ & $24.76 \pm 5.91 *$ & $26.90 \pm 4.70^{*}$ & $27.43 \pm 4.17 *$ \\
\hline & $\mathrm{AA}$ & $14.35 \pm 4.03$ & $25.16 \pm 4.47^{*}$ & $24.72 \pm 5.74^{*}$ & $24.66 \pm 4.07^{*}$ & $24.46 \pm 4.46^{*}$ & $24.11 \pm 3.86^{*}$ \\
\hline & AS & $15.43 \pm 3.75$ & $24.68 \pm 8.58^{*}$ & $24.46 \pm 5.53^{*}$ & $26.73 \pm 6.01 *$ & $24.69 \pm 4.69 *$ & $26.01 \pm 5.04 *$ \\
\hline & A & $15.18 \pm 4.65$ & $26.59 \pm 5.11^{*}$ & $25.58 \pm 5.08^{*}$ & $26.48 \pm 4.13 *$ & $27.40 \pm 4.15^{*}$ & $25.18 \pm 5.19^{*}$ \\
\hline
\end{tabular}

The table strain values are negative; BA: at the base of the front wall, BAS: near to the wall of the base next door, MA: central anterior, MAS: central near to intramural next door, AA: apex anterior, AS: inter-apical next door, A: apex; compared with group $\mathrm{A},{ }^{*} \mathrm{P}<0.05$. 


\section{DISCUSSION}

AMI is the sudden interruption of coronary blood flow due to plaque rupture and acute thrombosis, resulting in acute myocardial ischemia that leads to severe and lasting necrosis of the corresponding myocardium. With increased and improved means of early AMI reperfusion therapy, acute mortality has dropped substantially. However, the incidence of heart failure after AMI and its resultant mortality has increased significantly.

The occurrence of heart failure after AMI is closely related to microcirculation, vasospasm, the renin-angiotensin-aldosterone system (RAAS), and sympathetic nervous system activation (Di Somma et al., 2008). Exogenous rhBNP is a 17-cyclic polypeptide consisting of 32 amino acid residues. Via binding to its receptor, rhBNP can cause a variety of physiological effects including natriuresis, diuresis, blood vessel dilation, pulmonary artery wedge pressure reduction, coronary artery expansion, coronary blood flow increase, enhanced anti-myocardial ischemia, and hypoxia capacity. It can inhibit activity of the sympathetic nervous system and RAAS, reduce myocardial fibrosis, inhibit ventricular remodeling, and suppress thrombosis via reducing vascular congestion and coronary spasm (Peacock et al., 2004; Martin et al., 2008), thereby improving patient prognosis. Emerman (2002) found that the direct cause of approximately $14 \%$ patients with acute decompensated chronic heart failure was severe myocardial ischemia caused by acute coronary syndrome. In elderly patients, the proportion of patients with acute coronary syndrome caused by acute decompensated heart failure may be higher. A recent study (Peacock et al., 2005) showed that for patients with acute decompensated heart failure caused by acute coronary syndrome or a variety of other reasons, including acute deterioration of heart function, rhBNP treatment can improve heart function and reduce the incidence of major adverse cardiac events. Clinical studies have focused on the impact of rhBNP application on cardiac function in patients with acute coronary syndrome; however, follow-up studies of left ventricular function in specific local segments are lacking. In the present study, left ventricular function was assessed with echocardiography using the latest index (myocardial variables) to evaluate the impact of rhBNP on left ventricular local segmental function in patients with acute anterior myocardial infarction. Via 3DSTI technology, the three-dimensional motion trajectory of each myocardial spot was continuously tracked in the heart. Real-time variable curves of different cardiac tissues for different movement directions were obtained, and when compared with two-dimensional ultrasound speckle tracking imaging technology, left ventricular systolic function was more realistically and accurately reflected (Pérez et al., 2009).

In our study, 3D-STI technology was used to investigate the impact of rhBNP intervention on left ventricular function and local segmental motion in acute anterior or extensive anterior ST-segment elevation myocardial infarction. The results showed that within $6 \mathrm{~h}$ of admission, EF values in each group were not statistically significantly different; 1 week after PCI, EF values continued to decline in Group A, and EF values in Groups B and C did not show significant change. LVEDV and LVESV showed the same trends. Treatment with rhBNP was administered to patients in Groups B and C within 6 hours of admission, and BA, BAS, MA, MAS, AA, AS, and A segments in these 2 groups showed significant increase in function compared with those in group A. The changed segments were in line with the blood supply distribution of the left anterior descending artery and are the segments that would be pathologically affected by an anterior myocardial 
infarction. In each group and at each time point, BIL, BAL, MIL, MAL, and AL segments supplied by the left circumflex artery and BIS, BI, MIS, MI, and AI segments supplied by the right coronary artery did not show significant change. These results suggest that although reperfusion therapy was performed within the appropriate time window, the resilience of the left ventricular myocardium significantly decreased, as expressed by lower systolic functionality and greater end-diastolic volume. Intervention with rhBNP can improve the resilience of local myocardium, thereby improving the mechanical function of the left ventricle and myocardial remodeling. Within $6 \mathrm{~h}$ after admission, rhBNP application showed no significant difference in heart function improvement and myocardial remodeling inhibition. This suggests that clinical hemodynamic stability and benefit to patients depends on the timing of medicine administration. Other studies have confirmed (Mignot et al., 2010; Thebault et al., 2011) that in 3D-STI quantitative evaluation of left ventricular wall systolic function, area was a more sensitive indicator. Our results showed that area variables in Group A were significantly different from those in Groups B and C, which supports this view.

Many studies have confirmed that rhBNP can improve the prognosis of patients with acute myocardial infarction, but most of the studies were limited to improvement of exercise tolerance, heart function, and other general aspects. Because effective assessment tools have previously been lacking, specific effects of rhBNP on myocardial segments were unknown. However, the recent development of 3D-STI techniques, with good reproducibility, non-invasiveness, and no requirement for a special operator, has allowed for the collection and simultaneous display of three-dimensional graphics of the heart, including real-time dynamic display and accurate quantitative measurements of the heart. Compared with conventional echocardiography, cardiac MRI, and other means of inspection, 3D-STI has unique advantages in the evaluation of cardiac function and structure.

In the present study, 3D-STI was used to investigate the segmental motion of the heart and obtain relevant data. However, 3D-STI technology necessitates higher collection requirements to ensure accuracy of the data, and because of poor image data, some results were missing in some patients. In addition, although 3D-STI technology overcomes the limitation of "lost track when crossing the flat" of 2D-STI technology (Crosby et al., 2009), the abovementioned acquisition process is still affected by various objective and subjective factors. The experimental data will inevitably have some deviation, and probe and analysis software need to be further improved.

In our study, although reperfusion therapy was performed within the appropriate time window, resilience of the left ventricular myocardium significantly decreased, and lower systolic functionality and greater end-diastolic volume were observed. Intervention with rhBNP can improve the resilience of local myocardium and thereby improve mechanical function of the left ventricle and myocardial remodeling. However, because of the small sample size and limited follow-up time of our study, the specific mechanism of rhBNP efficacy in patients with acute anterior myocardial infarction needs further clinical and experimental studies.

\section{ACKNOWLEDGMENTS}

Research supported by the non-directly Affiliated Hospital of Fujian Medical University Research Development Special Fund (\#FZS13023Y). 


\section{REFERENCES}

Crosby J, Amundsen BH, Hergum T, Remme EW, et al. (2009). 3-D speckle tracking for assessment of regional left ventricular function. Ultrasound Med. Biol. 35: 458-471.

Di Somma S, Magrini L, Tabacco F, Marino R, et al. (2008). Brain natriuretic peptide and N-terminal pro-B-type natriuretic peptide show a different profile in response to acute decompensated heart failure treatment. Congest Heart Fail. 14: 245-250.

Emerman CL (2002). Safety and efficacy of nesiritide for the treatment of decompensated heart failure. Rev. Cardiovasc. Med. 3 (Suppl 4): S28-34.

Helle-Valle T, Crosby J, Edvardsen T, Lyseggen E, et al. (2005). New noninvasive method for assessment of left ventricular rotation: speckle tracking echocardiography. Circulation 112: 3149-3156.

Martin FL, Chen HH, Cataliotti A and Burnett JC Jr. (2008). B-type natriuretic peptide: beyond a diagnostic. Heart Fail Clin. 4: 449-454.

Mignot A, Donal E, Zaroui A, Reant P, et al. (2010). Global longitudinal strain as a major predictor of cardiac events in patients with depressed left ventricular function: a multicenter study. J. Am. Soc. Echocardiogr. 23:1019-1024.

Peacock WF 4th, Emerman CL and Young J (2004). Nesiritide in congestive heart failure associated with acute coronary syndromes: a pilot study of safety and efficacy. J. Card. Fail. 10: 120-125.

Peacock WF, Emerman CL and Silver MA (2005). Nesiritide added to standard care favorably reduces systolic blood pressure compared with standard care alone in patients with acute decompensated heart failure. Am. J. Emerg. Med. 23: 327-331.

Pérez de Isla L, Balcones DV, Fernández-Golfín C, Marcos-Alberca P, et al. (2009). Three-dimensional-wall motion tracking: a new and faster tool for myocardial strain assessment: comparison with two-dimensional-wall motion tracking. J. Am. Soc. Echocardiogr. 22: 325-330.

Thebault C, Donal E, Bernard A, Moreau O, et al. (2011). Real-time three-dimensional speckle tracking echocardiography: a novel technique to quantify global left ventricular mechanical dyssynchrony. Eur. J. Echocardiogr. 12: 26-32. 